\section{Ultrasonography of the ankle joint}

\author{
Jung Won Park', Sun Joo Lee', Hye Jung Choo', Sung Kwan Kim¹, Heui-Chul Gwak², \\ Sung-Moon Lee ${ }^{3}$
}

Departments of ${ }^{1}$ Radiology and ${ }^{2}$ Orthopedic Surgery, Inje University Busan Paik Hospital, Inje University College of Medicine, Busan; ${ }^{3}$ Department of Radiology, Dae Kyung Imaging Center, Daegu, Korea

Ankle disorders are a relatively common pathological condition, and ankle injuries account for approximately $14 \%$ of sports-related orthopedic emergency visits. Various imaging modalities can be used to make a diagnosis in cases of ankle pain; however, ultrasound (US) has several benefits for the evaluation of ankle pain, especially in the tendons, ligaments, and nerves of the ankle. The purpose of this article is to review the common causes of ankle pathology, with particular reference to US features. In addition, the importance of a dynamic evaluation and a stress test with US is emphasized.

Keywords: Ultrasonography; Ankle; Anatomy; Tendons; Ligaments; Nerves

\section{Introduction}

Ankle injuries account for approximately $14 \%$ of sports-related orthopedic emergency visits [1]. Various pathological conditions can affect the ankle, including trauma, overuse disorders, and inflammatory conditions [2]. Several imaging modalities, such as computed tomography, magnetic resonance imaging, and ultrasound (US) can be used to evaluate the ankle. However, US has several benefits for the evaluation of the tendons and ligaments of the ankle, such as its suitability to be integrated with a dynamic assessment and a stress test, its ability to provide real-time analysis, its avoidance of the risk of radiation exposure, and its cost-effectiveness [3]. US is especially powerful when used to evaluate a tear, subluxation, or dislocation in a dynamic examination and when performing a comparison with the contralateral extremity [4]. Dynamic imaging with muscle contraction or passive movement is often helpful. Additionally, Doppler imaging may be used to distinguish small intrasubstance tears from blood vessels that can occur in a tendinopathic tendon [5].

In this article, we present findings regarding the use of US for various pathological conditions of the ankle. Such conditions can be classified in terms of the anatomical divisions of the ankle, such as the tendons, ligaments, nerves, and bones. The relationships of the anatomy of the ankle with US examination results have been studied previously; therefore, we focused on the ultrasonographic features of common pathological entities of the tendons, ligaments, and nerves of the ankle.

\section{Normal Anatomy and Technique of US Examination}

The ankle tendon can be divided into four anatomic groups: medial, lateral, anterior, and posterior

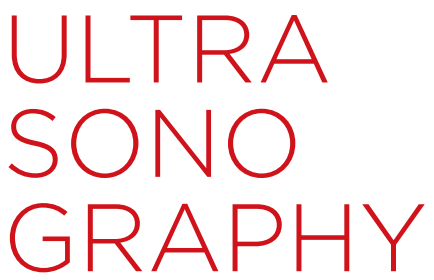

\section{REVIEW ARTICLE}

https://doi.org/10.14366/usg. 17008 pISSN: 2288-5919 • elSSN: 2288-5943 Ultrasonography 2017;36:321-335

Received: February 7, 2017

Revised: April 3, 2017

Accepted: April 5, 2017

Correspondence to:

Sun Joo Lee, MD, Department of Radiology, Inje University Busan Paik Hospital, Inje University College of Medicine, 75 Bokji-ro, Busanjin-gu, Busan 47392, Korea

Tel. +82-51-890-6579

Fax. +82-51-896-1085

E-mail: sunjulee98@naver.com

\begin{abstract}
This is an Open Access article distributed under the terms of the Creative Commons Attribution NonCommercial License (http://creativecommons.org/ licenses/by-nc/3.0/) which permits unrestricted noncommercial use, distribution, and reproduction in any medium, provided the original work is properly cited.
\end{abstract}

Copyright (C) 2017 Korean Society of Ultrasound in Medicine (KSUM)

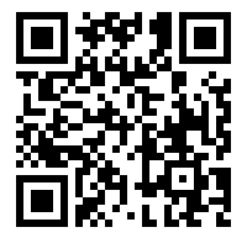

How to cite this article: Park JW, Lee SJ, Choo HJ, Kim SK, Gwak HC, Lee SM. Ultrasonography of the ankle joint. Ultrasonography. 2017 Oct;36(4)321-335. 
[3]. Each anatomic position contains different types of tendons, such as the tibialis posterior, flexor digitorum longus, and flexor hallucis longus in the medial group; the peroneus longus and brevis in the lateral group; the tibialis anterior, extensor hallucis longus, and extensor digitorum longus in the anterior group; and the Achilles and plantaris in the posterior group. These tendons are frequently oriented longitudinally and are best depicted in the transverse plane [6].

The ligaments of the ankle are composed of three ligamentous groups: the syndesmotic ligamentous complex, the lateral ligamentous complex, and the deltoid ligament.

Routine US examination of the ankle is performed in different positions that allow easy assessment of the medial, lateral, anterior, and posterior aspects.

\section{Medial}

The tendons of the medial ankle are the tibialis posterior tendon, the flexor digitorum longus tendon, and the flexor hallucis longus tendon, which run in the anteroposterior direction.

Transverse images obtained posterior to the medial malleolus, posterior tibialis tendon, and flexor digitorum longus are detected below the flexor retinaculum. Moving the transducer to a more posterior position allows visualization of the flexor hallucis longus tendon between the medial and lateral tubercles of the posterior talar process [3].

The medial deltoid ligament has a triangular or fan shape and originates from the apex of the medial malleolus. It is divided into a deep layer and a superficial layer. The deep layer contains the anterior tibiotalar ligament and posterior tibiotalar ligament, while the superficial layer contains the tibionavicular ligament, tibiospring ligament, and tibiocalcaneal ligament [3]. The posterior tibiotalar ligament is the thickest of the medial ligaments of the ankle. When this region is evaluated using US, the transducer is placed on the tip of the medial malleolus and is then moved posteriorly to the inferior end of the heel in an oblique longitudinal direction. The posterior tibiotalar ligament can be identified by its thick, striated appearance.

\section{Lateral}

The peroneus longus muscle arises from the lateral condyle of the tibia and the proximal fibula, and it inserts on the plantar surface of the medial cuneiform bone and the proximal first metatarsal bone. The peroneus brevis muscle originates more distally, from the distal two-thirds of the lateral fibular shaft, and inserts on the lateral aspect of the base of the fifth metatarsal bone [7].

Transverse images are obtained at the supramalleolar, malleolar, and inframalleolar segments while tilting the transducer perpendicular to the tendons.
At the supramalleolar level, the peroneus brevis tendon is visualized as a hypoechoic structure located between the peroneus longus tendon and the bone cortex. At the malleolar level, US can differentiate the two peroneal tendons under the superior peroneal retinaculum with the peroneus longus muscle superiorly. In the inframalleolar area, the peroneus longus tendon crosses under the peroneal tubercle, and the peroneus brevis tendon is located above the peroneal tubercle.

The ligaments of the lateral ankle consist of the lateral ligamentous complex, the anterior talofibular ligament (ATFL), posterior talofibular ligament (PTFL), and calcaneofibular ligament (CFL) [8].

The ATFL runs anteriorly from the lateral malleolus and attaches to the lateral side of the talar neck. The ATFL is the most vulnerable ligament to inversion injuries. On a transverse scan anterior to the lateral malleolus, the ATFL can be identified by its straight and striated appearance.

The CFL runs inferiorly from the lateral malleolus to the posterolateral aspect of the calcaneus. For scanning of the CFL, the transducer is placed between the lateral malleolus and the posterolateral aspect of the calcaneus, and the CFL exhibits a curved course with hypoechogenicity. The CFL can be well depicted during dorsiflexion of the ankle on US.

The PTFL originates from the posterior aspect of the lateral malleolus and inserts into the lateral tubercle of the posterior talar process. However, it is difficult to evaluate the PTFL by US due to its deep location [9].

\section{Anterior}

When examining of the anterior tendon of the ankle, obtaining axial images can allow several tendons to be visualized in a single image and differentiated. The tibialis anterior tendon (TA) is located at the anteromedial aspect of the ankle. It descends obliquely to insert into the medial aspect of the first cuneiform. The extensor hallucis longus tendon (EHL) runs parallel and lateral to the TA, and the extensor digitorum longus tendon (EDL) is found in a more lateral position.

The anterior tibial artery is located below the anterior tendons and runs between the EHL and EDL. The deep peroneal nerve is located proximal to the medial aspect of the artery, and it crosses the artery distally to descend along its lateral border.

\section{Posterior}

The Achilles tendon is a conjoined tendon formed by the fusion of the distal tendon laminae of the soleus and gastrocnemius muscles. During its course, it rotates $90^{\circ}$ laterally, so that its soleus component inserts into the posteromedial aspect and its gastrocnemius component inserts into the posterolateral aspect of 
the calcaneus [3].

\section{Pathologies of the Tendon}

Pathological conditions of the ankle tendons include tendinosis, tenosynovitis, tears, subluxation, and dislocation. These conditions show similar ultrasonographic features elsewhere in the body [4].

Tendinosis results in thickening of the tendon, focal or diffuse areas of hypoechogenicity, and preservation of a fibrillar appearance (Fig. 1) [10]. It may occasionally be difficult to differentiate an area of tendinosis from a partial-thickness tear on US.

Tendinitis and tenosynovitis, which involve inflammation around the tendon or tendon sheath, are common tendon disorders of the ankle. The etiology of tendinitis and tenosynovitis is thought to be secondary to trauma; repetitive local stress; systemic joint disorders, such as rheumatoid arthritis or ankylosing spondylitis; or infection [10]. Tenosynovitis and tendinitis appear as irregularly thickened and hypoechoic synovial membranes or tendons. These conditions are usually accompanied by fluid in the tendon sheath or synovial fluid, which appears as a "target sign" in the axial plane and a "rail-track" in the longitudinal plane [11]. Color Doppler can help to determine the degree of activity of the inflammation of the tendon sheath inside the synovium or tendons (Fig. 2).

A tendon tear may be seen as a hypoechoic or anechoic gap in the fibrillar-patterned tendon with retraction of the tendon.

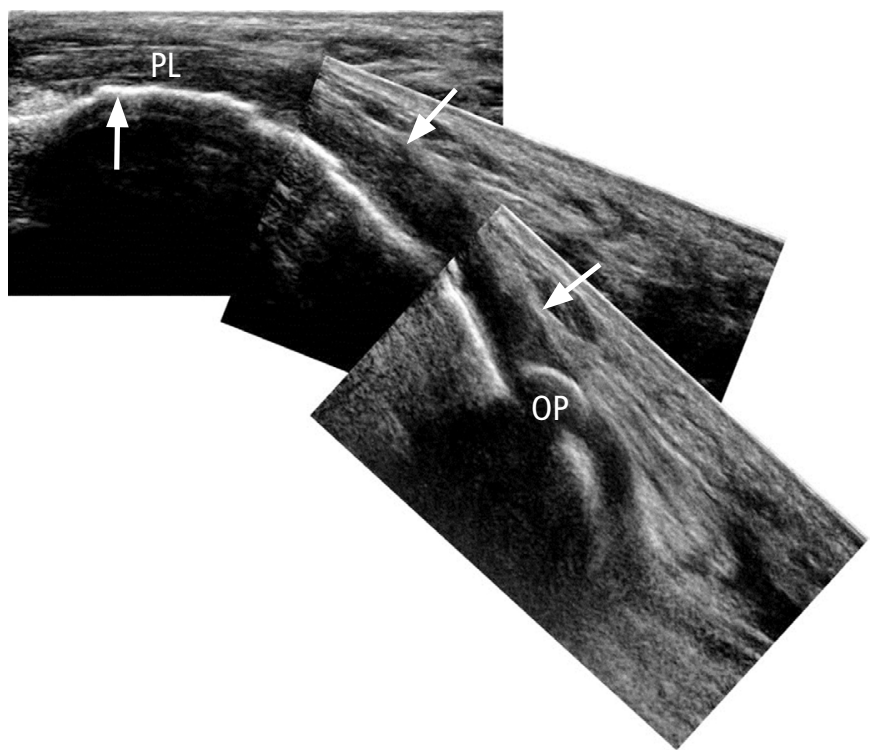

A

Fig. 1. Tendinosis of the peroneus longus tendon.

Long-axis (A) and short-axis (B) ultrasonography of the peroneus longus tendon (arrows) shows diffusely enlarged and hypoechoic swelling of the tendon without tendon fiber discontinuity. There is no evidence of synovial thickening or synovial fluid collection. PL, peroneus longus tendon; $\mathrm{PB}$, peroneus brevis tendon; $\mathrm{LM}$, lateral malleolus; $\mathrm{OP}$, os peroneale.

e-ultrasonography.org

Ultrasonography 36(4), October 2017 


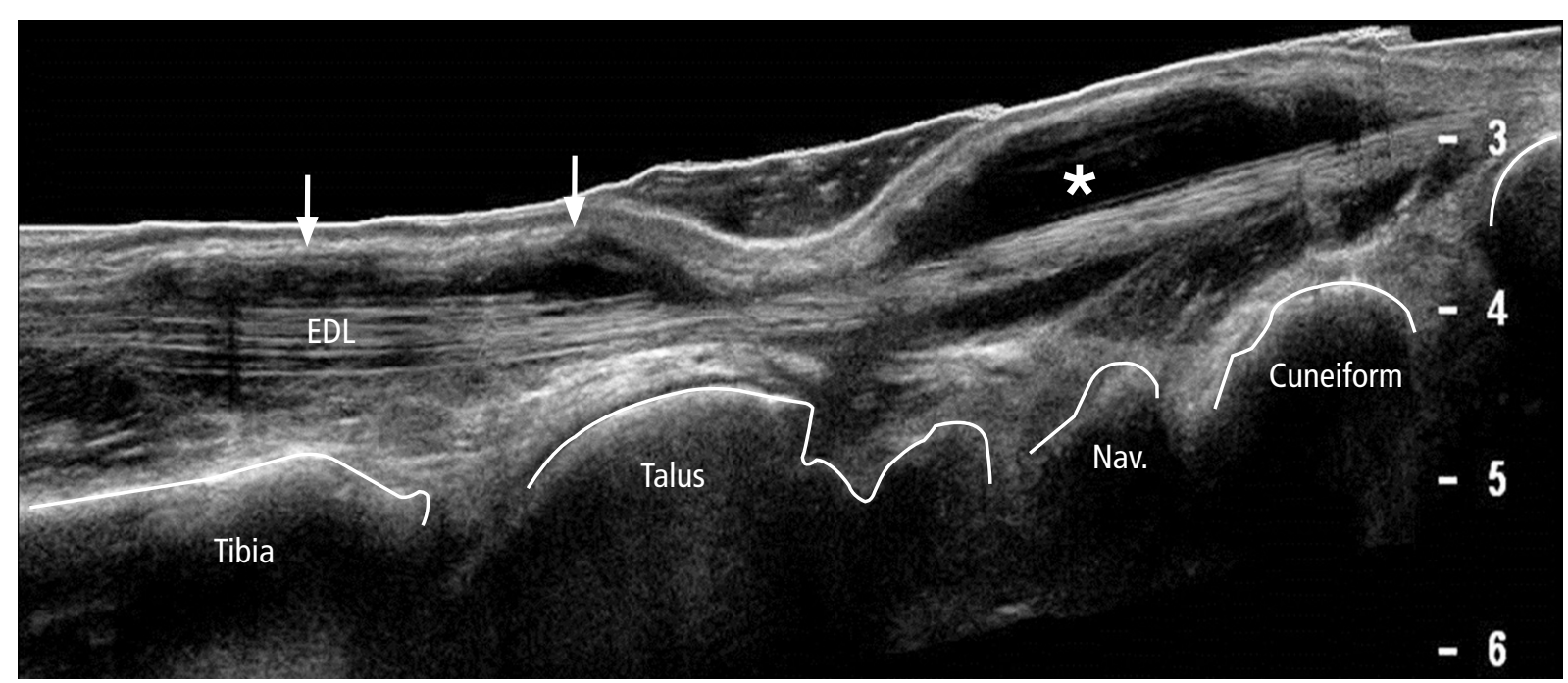

A

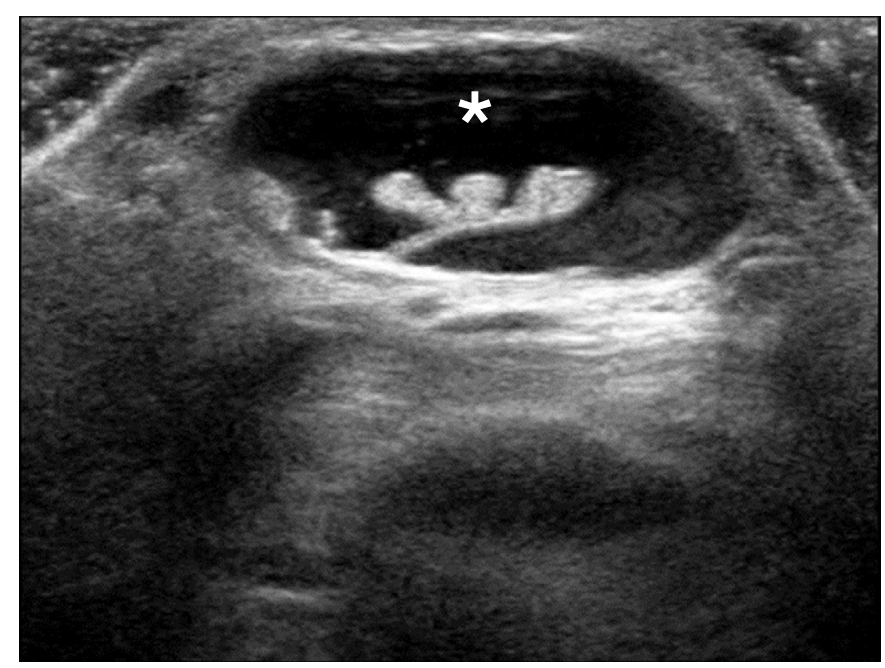

B

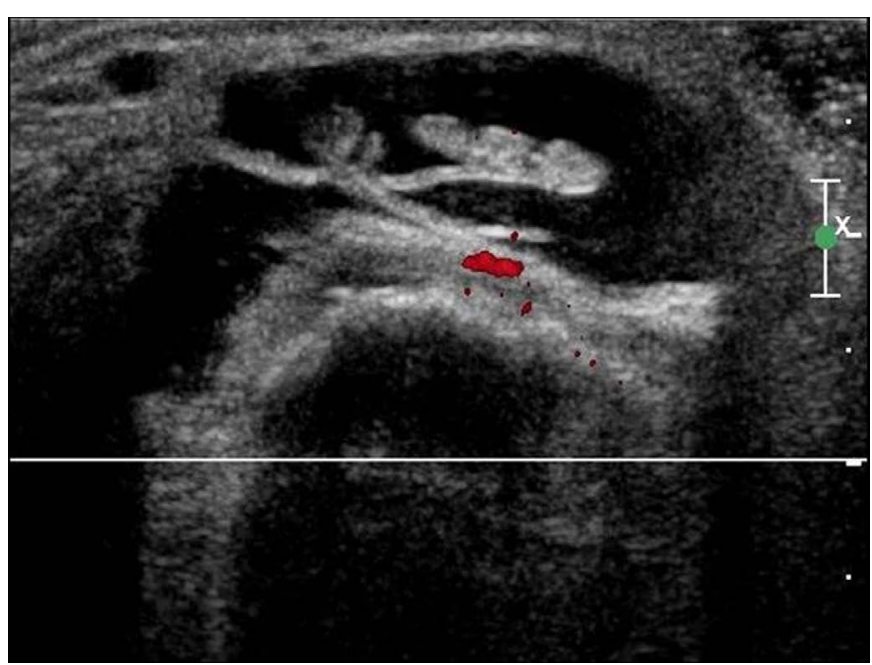

C

Fig. 2. Tenosynovitis of the extensor digitorum longus.

Long-axis (A) and short-axis (B) ultrasonography of the extensor digitorum longus shows anechoic distention of the tendon sheath (asterisks), synovial hypertrophy (arrows), and increased vascularity on the color Doppler image (C). EDL, extensor digitorum longus; Nav., navicular bone.

distinct hemi-tendons, irregularities of the tendon, or features of associated tenosynovitis (Fig. 3). A boomerang shape of the peroneus brevis tendon is likely to suggest the early stage of a longitudinal tear caused by weakening and thinning of the central part of the tendon [7].

Isolated peroneus longus tears are uncommon and mostly observed at the cuboid bone level. On US, a partial tear appears as thinned or thickened and presents an irregular hypoechoic internal appearance with loss of the normal fibrillar pattern. A full-thickness tear of the peroneus longus tendon is indicated by longitudinal splits or complete tendon discontinuity with retraction. Frequently, there is associated tenosynovitis [13].

Intrasheath subluxation of the peroneal tendons develops when the tendons reverse their anatomic location within the peroneal groove with an intact superior peroneal retinaculum (SPR) [7]. Intrasheath subluxation of the peroneal tendons was divided into two subtypes by Raikin et al. [16]. In type A, the tendons snap over one another and switch their relative positions within the peroneal groove without a tendon tear or disruption of the SPR. In type $B$, subluxation of the peroneus longus tendon occurs through a longitudinal split tear within the peroneus brevis tendon, which results in a portion of the longus tendon lying deep relative to the 
brevis tendon at this level [16]. Dynamic US examination reveals subluxation of the peroneus brevis and longus tendons over each other within the peroneal groove, with an intact SPR while dorsiflexion and an eversion maneuver are performed (Fig. 4).

Peroneal tendon dislocation occurs when one or both tendons become displaced from the retromalleolar groove (Fig. 5), often only transiently during ankle movement or muscle contractions [17]. This is commonly associated with an SPR injury.

The SPR is composed of fibrous retaining bands that bind down the peroneus longus and brevis tendons. An acute SPR

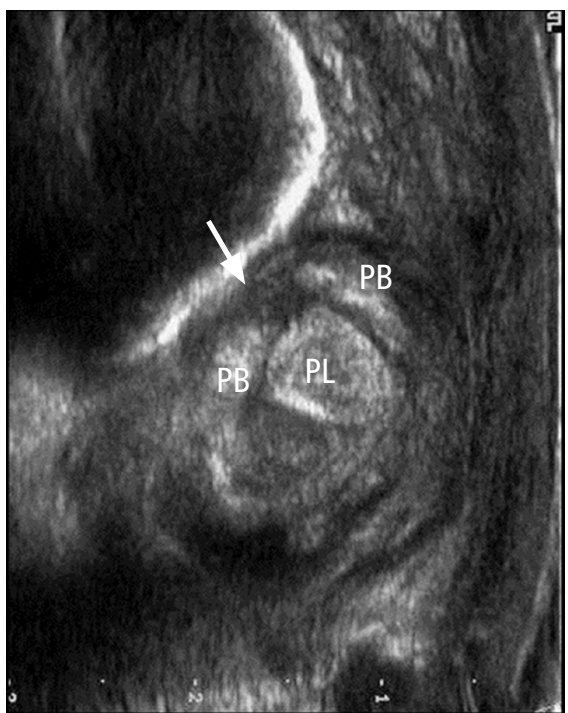

A

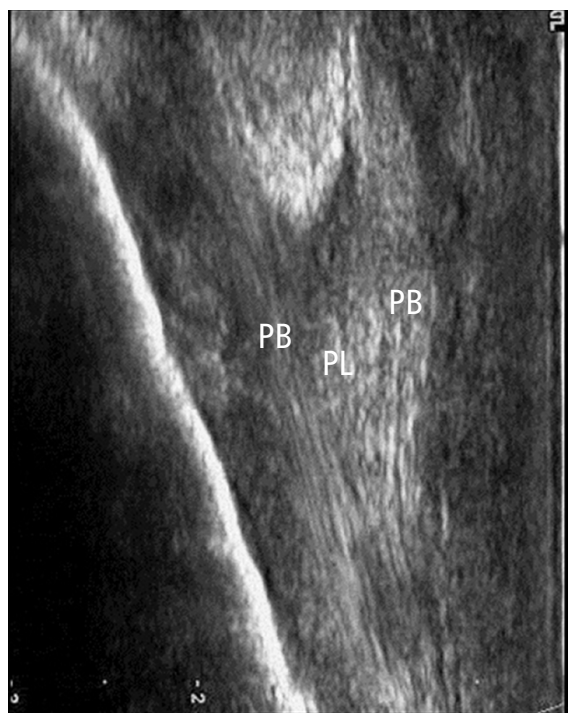

B

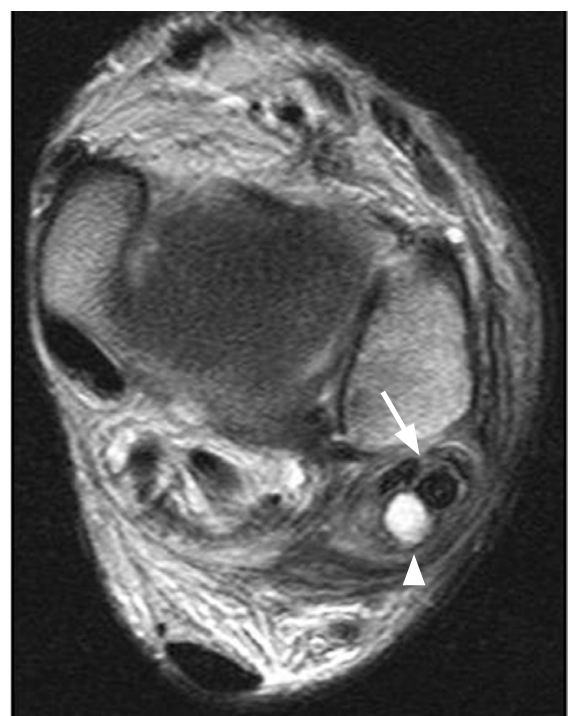

C

Fig. 3. Longitudinal tear of the peroneus brevis tendon.

Short-axis (A) and long-axis (B) ultrasonography scanned at the lateral malleolar area shows that the peroneus brevis tendon is split (arrow) and divided into two separate bundles of fibers. The peroneus longus tendon is intact. C. An axial T2-weighted magnetic resonance image reveals a bisected peroneus brevis tendon (arrow) and cystic lesion (arrowhead) posterior to the peroneus longus tendon. PB, peroneus brevis tendon; PL, peroneus longus tendon.

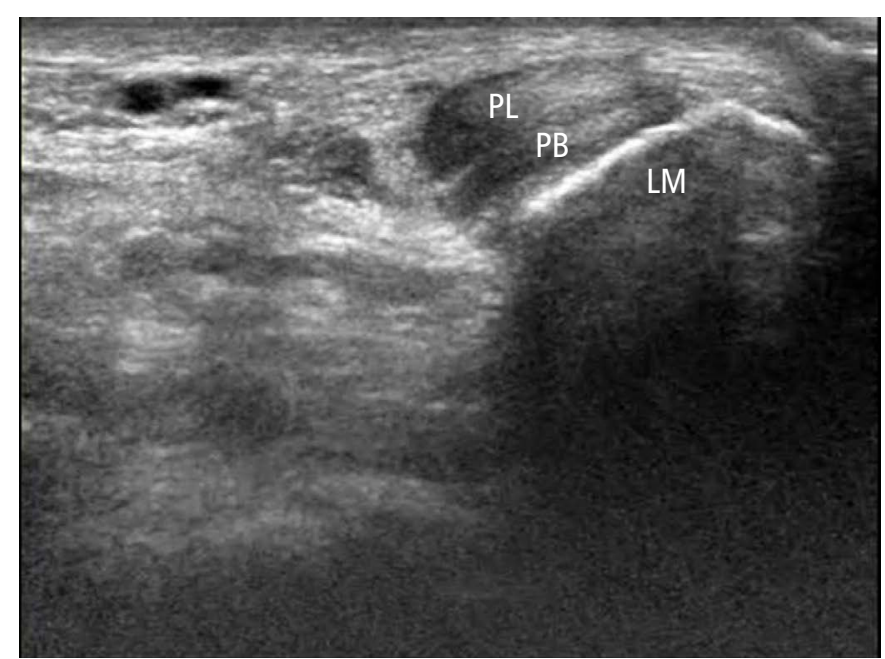

A

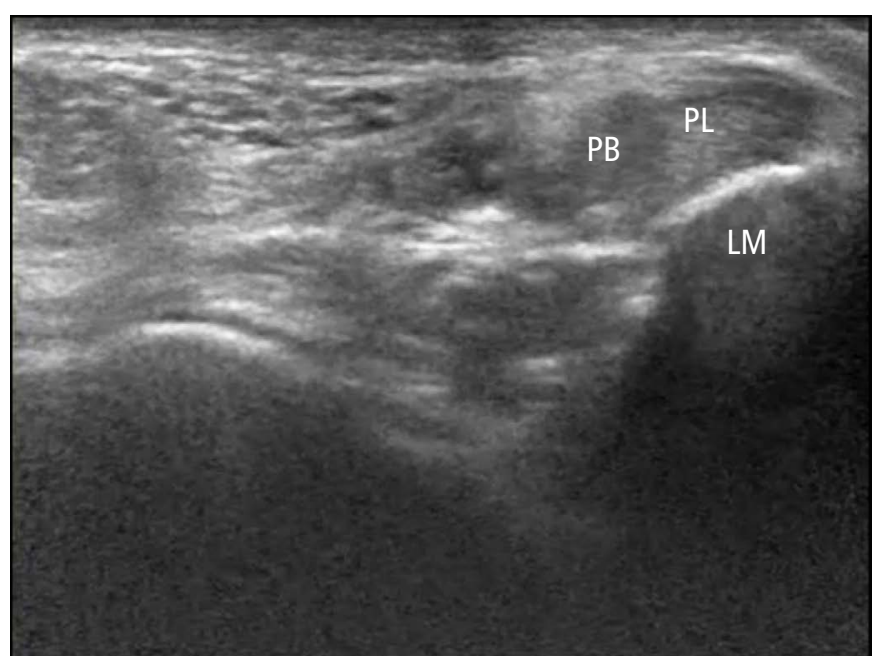

B

Fig. 4. Dynamic ultrasound of an intrasheath peroneal subluxation (Raikin type A).

Short-axis ultrasonography over the lateral malleolus is obtained at rest (A) and during dorsiflexion and eversion of the foot (B). A. The peroneus brevis and peroneus longus tendons lie posterior to the tip of the lateral malleolus. $\mathrm{B}$. The peroneus longus and peroneus brevis tendons have switched their positions. LM, lateral malleolus; $\mathrm{PB}$, peroneus brevis tendon; PL, peroneus longus tendon. 


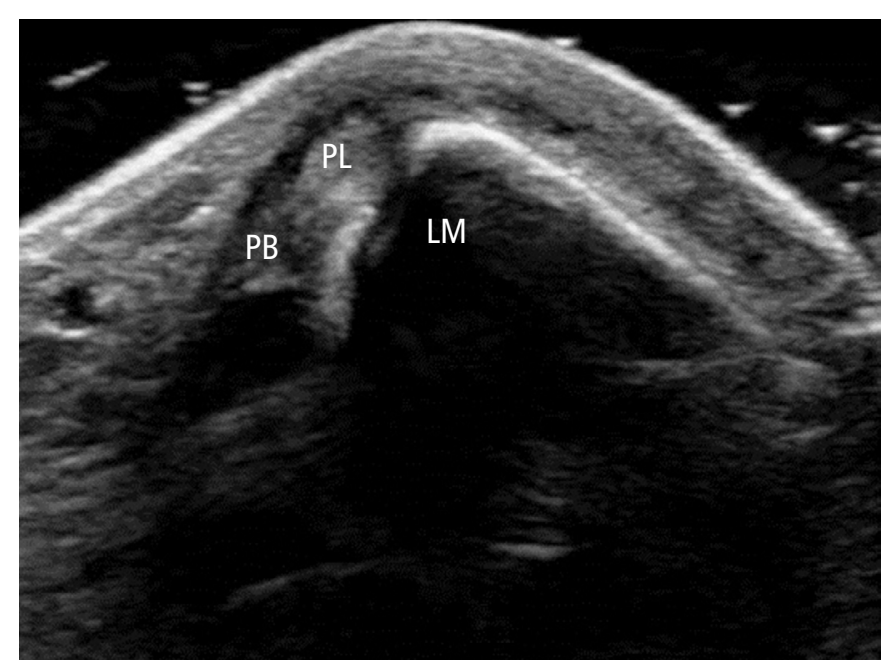

A

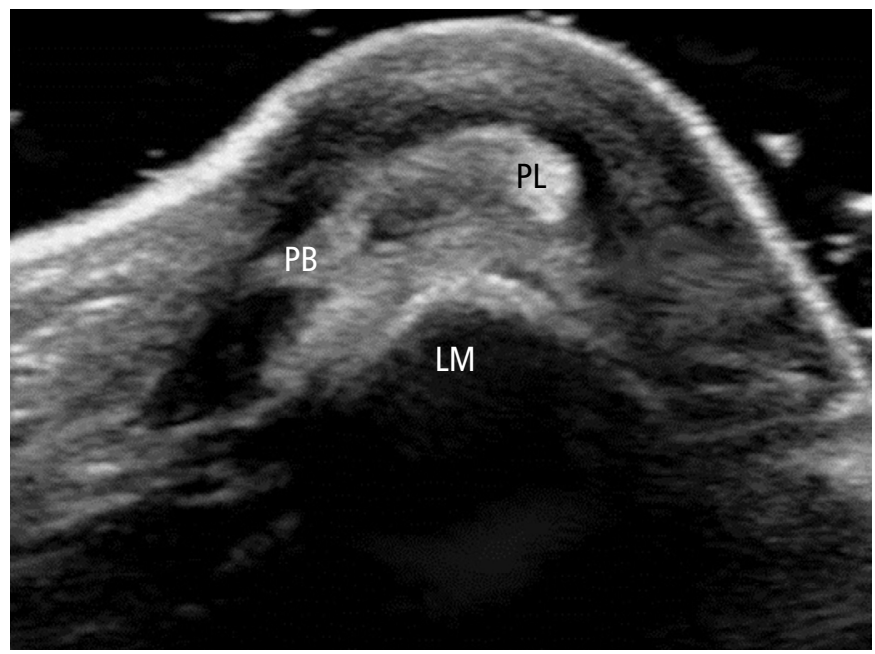

B

Fig. 5. Dynamic ultrasound of peroneal dislocation.

Short-axis ultrasonography over the lateral malleolus is obtained at rest (A) and during dorsiflexion and eversion of the foot (B). A. The neutral position of the peroneus brevis and peroneus longus tendon, which lies posterior to the tip of the lateral malleolus, can be seen. $B$. The peroneus longus and peroneus brevis tendons become displaced from the retromalleolar groove. LM, lateral malleolus; PB, peroneus brevis tendon; PL, peroneus longus tendon.

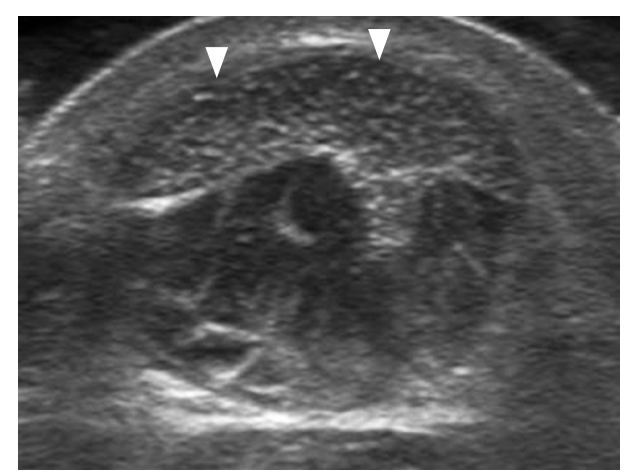

A

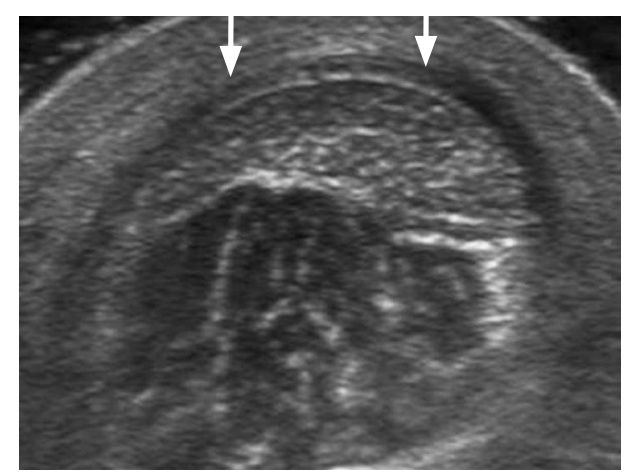

C

Fig. 6. Achilles paratendinopathy.

Short-axis (A) and long-axis (B) ultrasonography of the left Achilles tendon shows a normal appearance (arrowheads) of the Achilles tendon. Short-axis (C) and long-axis (D) ultrasonography of the right Achilles tendon shows a hypoechoic and thickened paratendon (arrows) around the Achilles tendon. The patient (26-year-old man) had right ankle pain just after running a marathon competition. 
injury manifests with ecchymosis, swelling, and pain at the lateral malleolus and may be mistaken for an ankle sprain. The most common mechanism of SPR injury involves the sudden contraction of the peroneal muscles either during an acute inversion injury to the dorsiflexed ankle or during forced dorsiflexion of the everted foot [18]. SPR injuries are graded using the Oden classification into four types [19]. In type I, the SPR and its periosteal attachment are elevated at the level of the fibular groove, forming a pouch. In type II injuries, there is a tear at the attachment of the SPR to the distal fibula. Type III injuries consist of an avulsion fracture at the attachment of the SPR to the distal fibula. In type IV injuries, there is a tear in the SPR at its posterior attachment [19].

\section{Achilles Tendon}

The Achilles tendon is the longest and strongest tendon in the body. An Achilles tendon injury is commonly caused by forced and sudden movement at the ankle joint, often occurring in active individuals in the third to sixth decades of life with no underlying history [20].

The Achilles tendon has a distinct anatomic structure compared to the other tendons of the ankle. It is not surrounded by a true synovial sheath; however, it is surrounded by a paratendon, which is composed of a single layer of cells (Fig. 6A, B) [21]. Therefore, instead of tenosynovitis, paratendinopathy can occur alone or in combination with the degeneration of this tendon [22].

Achilles tendinopathy appears as focal or diffuse thickening, with a mean thickness of $9 \mathrm{~mm}$ (a normal tendon measures 5 to 7 $\mathrm{mm}$ ) and a loss of anterior concavity in the transverse plane [23]. Achilles paratendinopathy usually reveals diffuse edema around the Achilles tendon (Fig. 6C, D). Small fluid collection can be seen in the retrocalcaneal bursa.

Partial and complete Achilles tendon ruptures are caused by acute muscle strain resulting from sudden or forceful dorsiflexion. The diagnosis of Achilles tendon rupture can depend on the patient's history and physical examination. Nevertheless, the initial diagnosis is missed in over $20 \%$ of cases [24]. On US, a focal defect with anechoic or hyperechoic hematoma can be seen, and the affected site is usually $2-6 \mathrm{~cm}$ from the calcaneal insertion site (Fig. 7). Therefore, the Achilles tendon should be scanned carefully from its calcaneal insertion to its myotendinous junction.

Haglund syndrome was first described in 1928 as retrocalcaneal bursitis associated with an abnormal protuberance of the posterosuperior border of the calcaneus [25]. It is caused by tendinosis, overuse, or

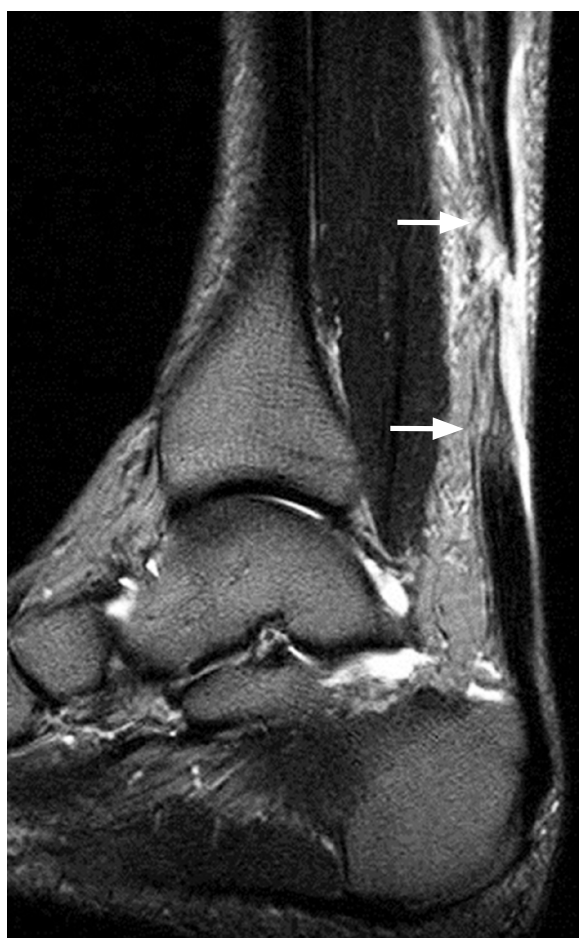

A

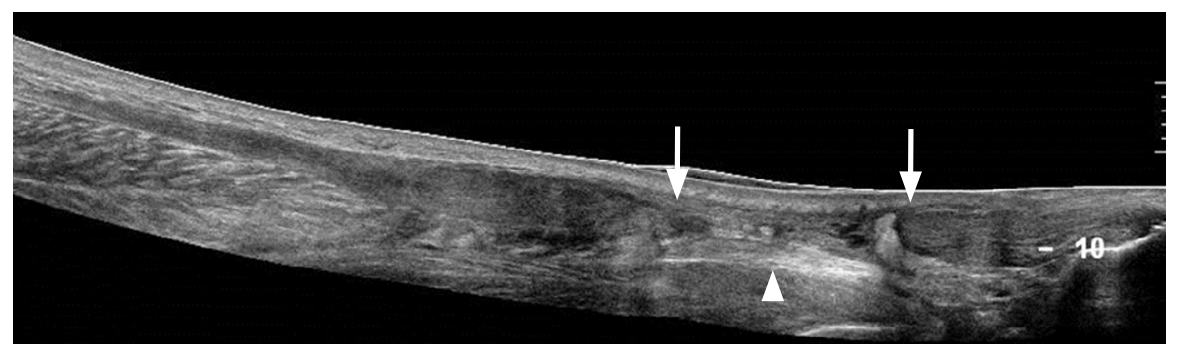

B

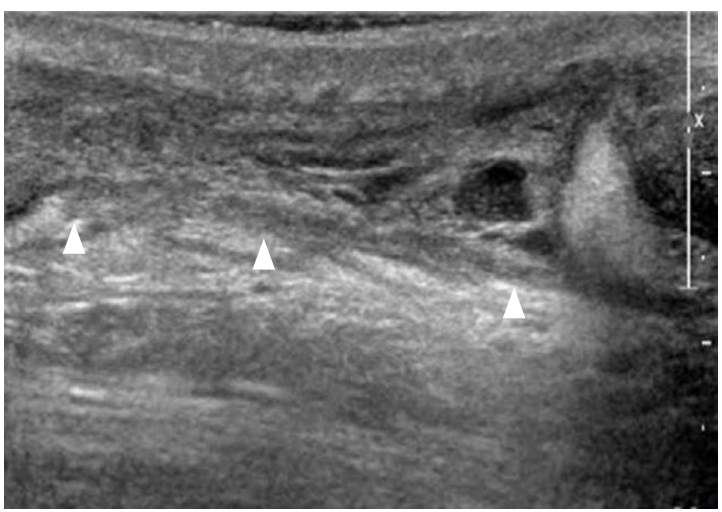

C

Fig. 7. Full-thickness tear of the Achilles tendon.

A. Sagittal magnetic resonance image of the Achilles tendon shows complete disruption of the tendon with two tendon stumps (arrows) and fluid collection at the gap. B, C. Long-axis ultrasonography of the Achilles tendon shows complete disruption (arrowheads) of the tendon fibers with two tendon stumps (arrows) with an irregular and retracted appearance. 
the wearing of an ill-fitting shoe that produces bone enlargement of the posterosuperior aspect of the calcaneus, leading to inflammation of the overlying tissues and Achilles tendon [8]. This abnormality is characterized by swelling of the superficial and retrocalcaneal bursa, Achilles tendinosis, and a prominent bursal projection of the calcaneus. On US, fusiform enlargement and inhomogeneity of the distal Achilles tendon with complex hypoechoic fluid in the retrocalcaneal bursa and thickening of the superficial calcaneal bursa are also present (Fig. 8) [26].
Achilles tendon xanthomas are painless soft tissue masses occurring most commonly in the distal one-third of the tendon, usually bilaterally and symmetrically. They are characterized by the localized accumulation of lipid-laden macrophages, inflammatory cells, and giant cells secondary to cholesterol deposition in the tissue. On US, an Achilles tendon xanthoma has a speckled or reticular appearance and extensive tendon thickening (Fig. 9) [27].

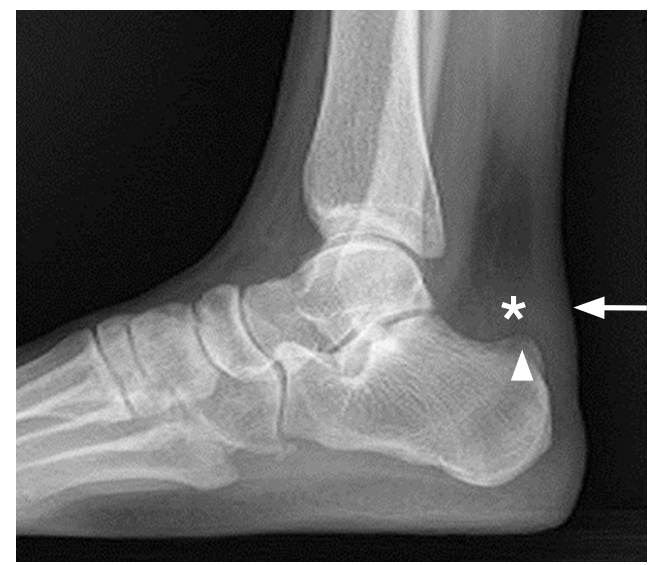

A

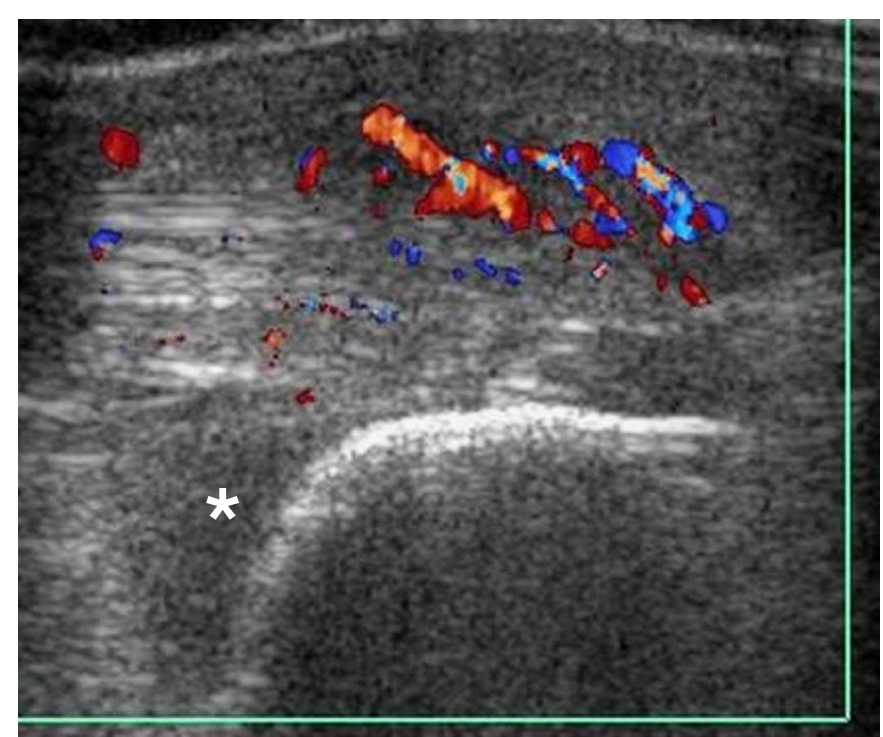

C

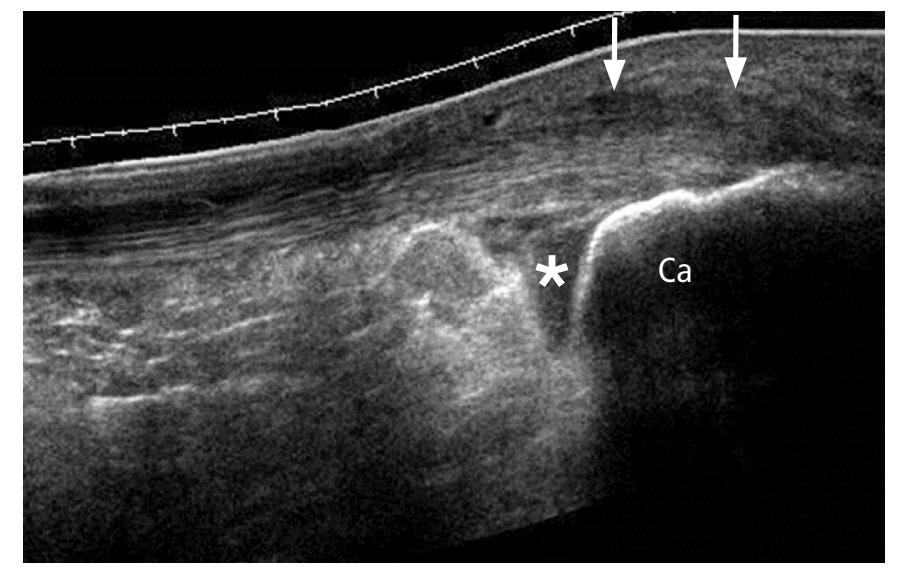

B

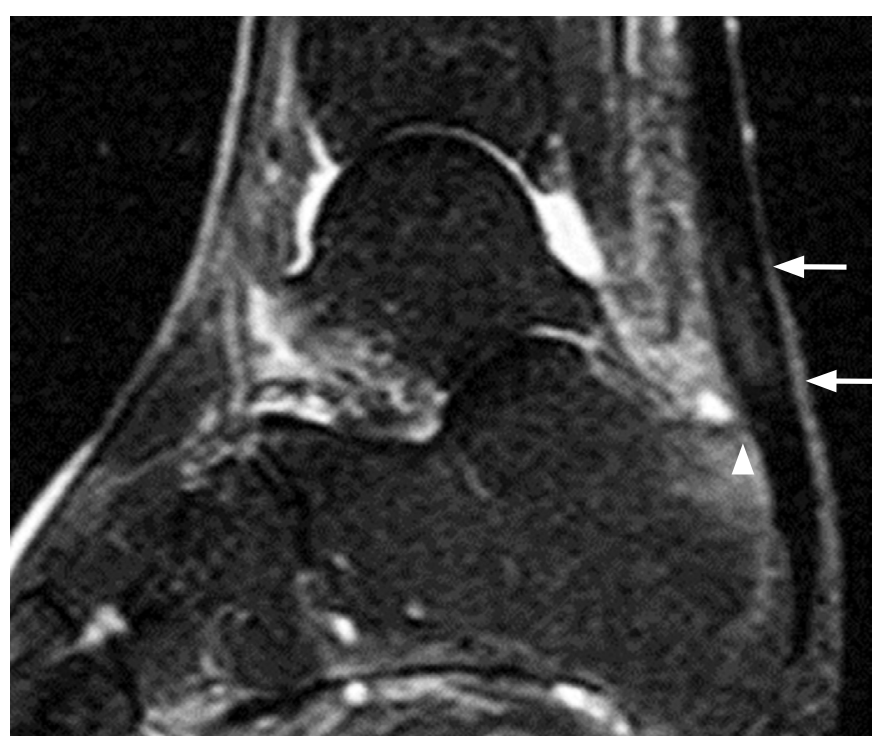

D

Fig. 8. Haglund syndrome of the Achilles tendon.

A. Lateral ankle radiograph shows bony hypertrophy of the posterosuperior aspect of the calcaneus (arrowhead), haziness in the Kager fat pad (asterisk), and swelling of the distal Achilles tendon (arrow). B, C. Long-axis (B) and color Doppler (C) ultrasonography of the distal Achilles tendon shows anechoic fluid collection in the retrocalcaneal bursa (asterisks), swelling with inhomogeneity in the distal Achilles tendon (arrows), and increased vascularity of the Achilles tendon. Ca, calcaneus. D. In a sagittal fat-suppressed T2-weighted magnetic resonance image, there is bony hypertrophy of the posterosuperior aspect of the calcaneus (arrowhead), fluid in the retrocalcaneal bursa, and swelling with internal increased signal intensity in the distal Achilles tendon (arrows). 


\section{Pathologies of the Ligament}

The ligament of the ankle is composed of three ligamentous groups: the syndesmotic ligamentous complex, the lateral ligamentous complex, and the deltoid ligament. On longitudinal scans, ligaments are well-defined linear structures with a fibrillar appearance that should not exceed $2 \mathrm{~mm}$ in thickness [28].

Morvan et al. [29] suggested a classification system based on US for ligamentous tears that involves three categories: mild, moderate (partial), and severe (complete). In mild acute sprains, the ligament may be normal or slightly thickened, and its hyperechoic fibrillar structure may be slightly altered. In a moderate (partial) tear, there is partial interruption with an anechoic area; however, the ligament remains tight during dynamic examination. In a severe (complete) ligamentous tear, there is complete interruption of fibers with an anechoic or hypoechoic gap, and the ligament may be wavy and does not tighten with dynamic stress (Figs. 10A, B, 1113). In chronic tears, US reveals thickening of the ligament, and ossifications may exist (Fig. 10D) [29].

\section{Anterior Talofibular Ligament}

The ATFL is the most injured ligament during inversion strains of the ankle, followed by the CFL [30]. Rupture of the ATFL and CFL together may occur in approximately 20\% of injuries [31].

The ATFL joins the anterior edge of the tip of the lateral malleolus [3]. To examine this ligament, a probe is placed in the transverse plane with the most distal aspect of the lateral malleolus in the longitudinal axis of the foot [2]. Dynamic tests, such as plantar

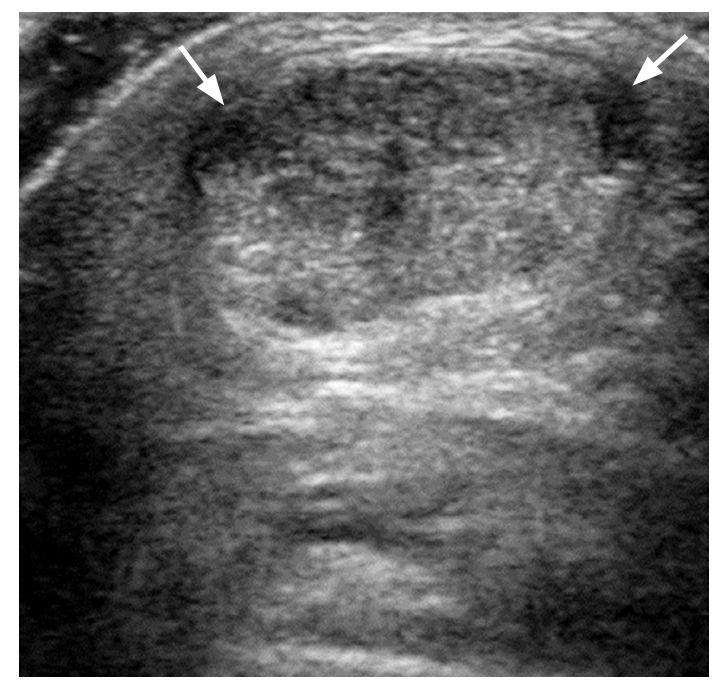

A

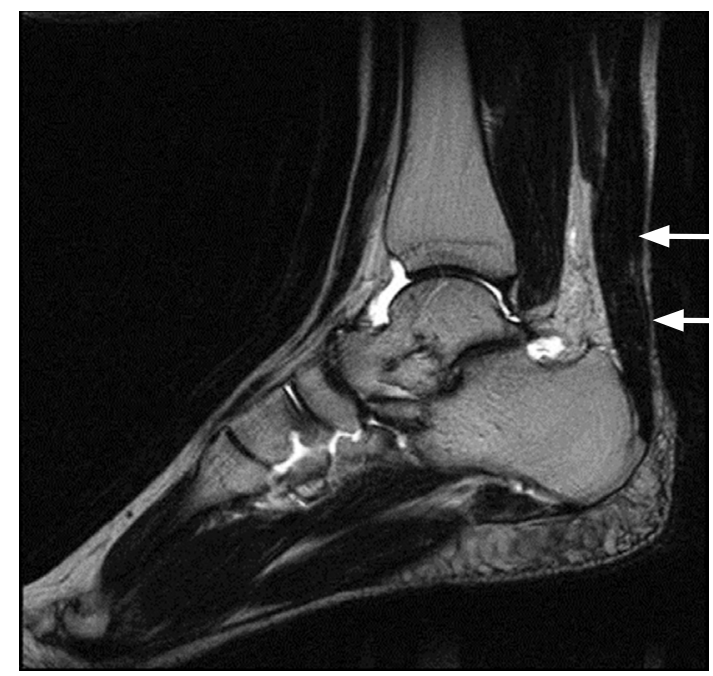

D

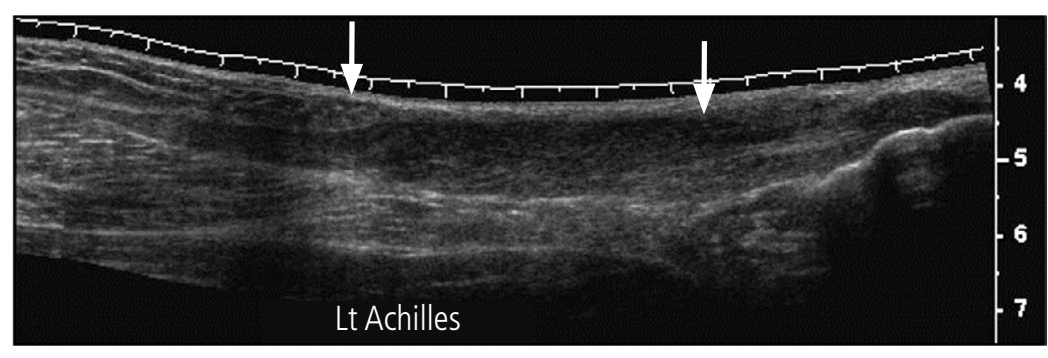

B

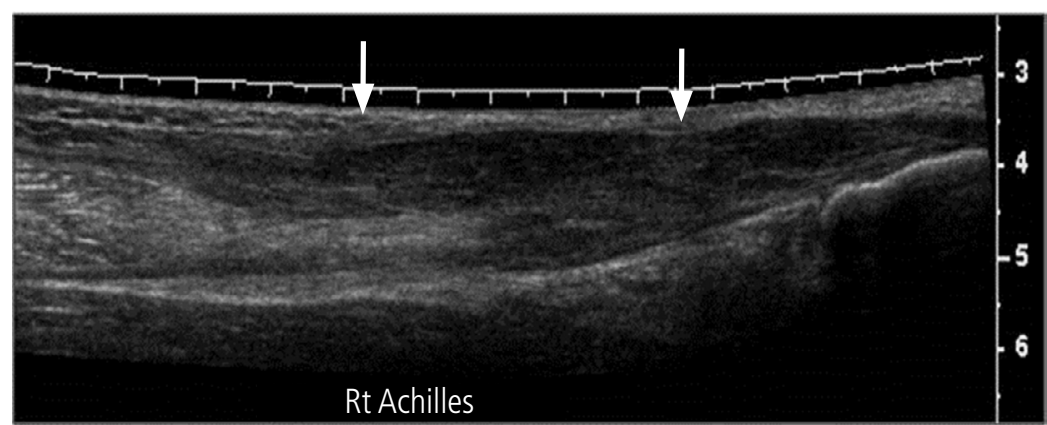

C

Fig. 9. Achilles tendon xanthoma.

A-C. Short-axis ( $A$ ) and long-axis ( $B$, left; $C$, right) ultrasonography of the Achilles tendon shows a heterogeneously enlarged tendon with convexity in the anterior aspect of the tendon and an internal reticular appearance (arrows). D. A sagittal T2-weighted magnetic resonance image shows extensive thickening of the Achilles tendon with convexity of the anterior aspect of tendon and a speckled appearance (arrows). 
flexion, inversion stress, or an anterior drawer test, may place tension on the ligament.

The ligament appears as a flattened hyperechoic structure located at the lower part of the lateral articular face of the talar dome (Fig. 10C).

\section{Posterior Talofibular Ligament}

The PTFL is composed of multiple thin bundles separated by fat, resembling the anterior cruciate ligament of the knee. It is hardly detectable with US because of its deep location [32].

\section{Calcaneofibular Ligament}

The CFL is usually sequentially torn after the ATFL during inversion sprains. In contrast, an isolated sprain is rare but may occur. Complete CFL tears are often accompanied by fluid in the peroneal tendon sheath [14]. When suspicious of an injury to the CFL, a stress inversion maneuver of the ankle joint and the anterior drawer test should be used to assess the integrity of the ATFL and CFL.

The CFL originates from the tip of the lateral malleolus; therefore, it is examined with coronal imaging by placing the upper angle of the transducer over the tip of the lateral malleolus (Fig. 11) [3]. The CFL can be well depicted during dorsiflexion of the ankle on US. This ligamentous structure lies deep in the peroneal tendons. It has been described as cylindrical and is typically $3-5 \mathrm{~mm}$ thick and $4-8 \mathrm{~mm}$ wide [33].

\section{Deltoid Ligament}

The strong deltoid ligament suffers eversion sprains less commonly than inversion sprains. The medial deltoid ligament has a triangular shape, originates from the apex of the medial malleolus, and fans over the internal aspect of the talus and calcaneus (Fig. 12).

This ligament is best shown during dorsiflexion and eversion stress on the hindfoot due to absence of anisotropy [28].

In acute tears of the deltoid ligament, the radiologist must look

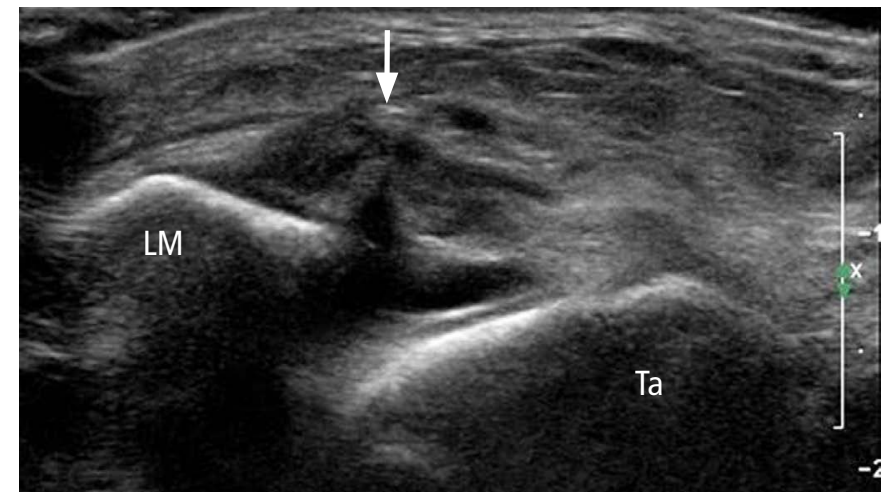

A

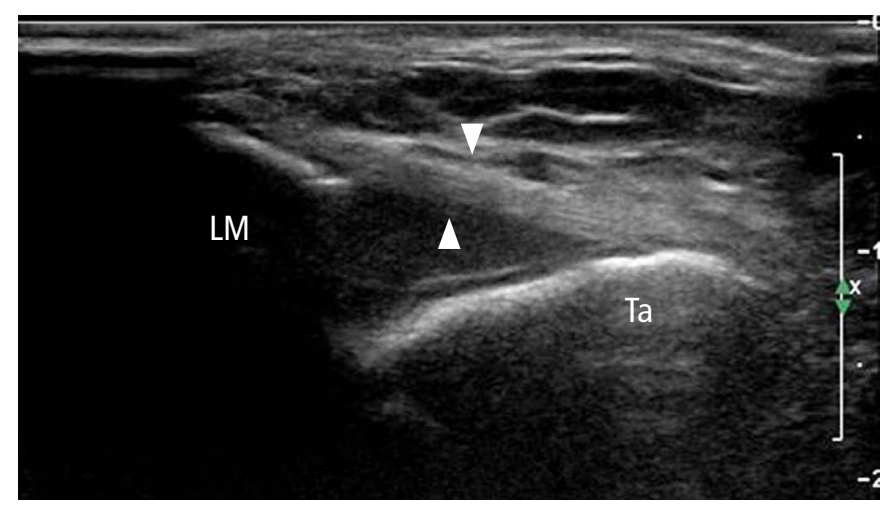

C

Fig. 10. Complete and partial tears of the ATFL.

A. Long-axis ultrasonography of the ATFL shows hypoechoic discontinuity of the ligament, compatible with a complete tear (arrow). B. An inversion stress image provides more diagnostic confidence regarding the complete tear (arrows). C. Normal appearance of the ATFL (arrowheads) is shown. D. In another patient with lateral ankle pain, the ligament exhibits heterogeneous hypoechogenicity with some calcifications. This finding reveals a chronic partial tear (arrow). ATFL, anterior talofibular ligament; LM, lateral malleolus; Ta, talus. 
for associated lateral malleolar fractures or distal tibiofibular tears [29].

\section{Anteroinferior Tibiofibular Ligament}

The anteroinferior tibiofibular ligament (AiTFL) starts from the distal tibia and descends obliquely between the adjacent margins of the tibia and fibula. Most commonly, injuries of the distal tibiofibular syndesmosis in ankle fractures occur in the AiTFL (83.8\%) on arthroscopic findings [34]. The AiTFL is the weakest of the syndesmotic ligaments and is the first to yield to forces that create an external rotation of the fibula around its longitudinal axis (Fig. $13 \mathrm{~A}, \mathrm{C})$. The AiTFL sometimes appears striated due to fat interposed between the fascicles of the ligament (Fig. 13B) [8].

\section{Pathologies of the Nerve}

\section{Tarsal Tunnel Syndrome}

Tarsal tunnel syndrome is an entrapment disorder of the tibial nerve and its branches on the medial side of the ankle. The clinical manifestations of tarsal tunnel syndrome include numbness and

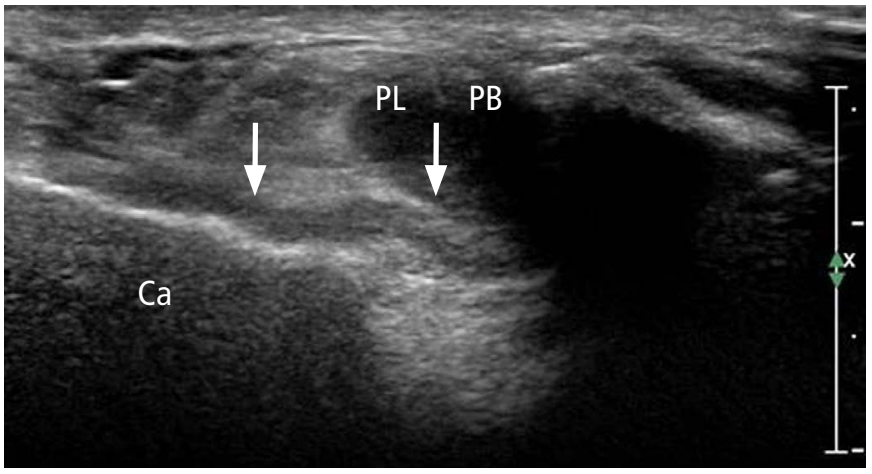

A

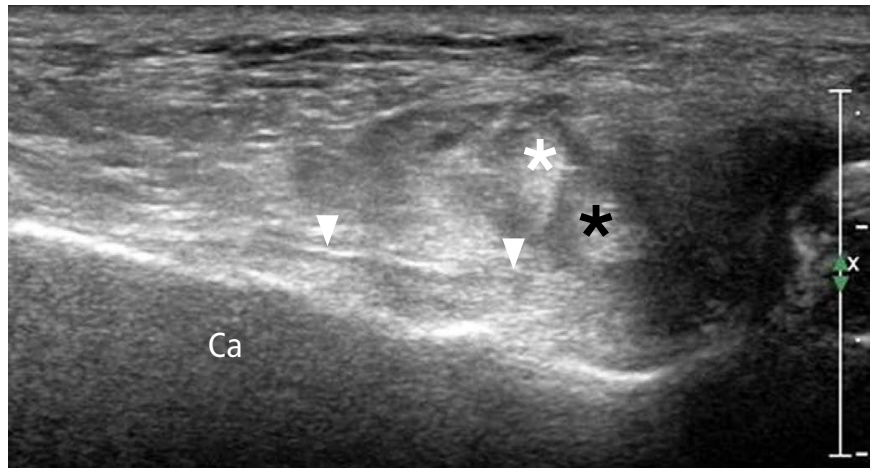

B

Fig. 11. Injury of the CFL.

A. Long-axis ultrasonography of the right CFL shows a hypoechoic and wavy appearance (arrows). The patient had an inversion injury of the ankle 1 day prior. B. Ultrasonography of the left side, with a normal CFL, shows a curved course with an echogenic fibrillar structure (arrowheads) located between the calcaneus and peroneal tendons. CFL, calcaneofibular ligament; PL, peroneus longus tendon (white asterisk); PB, peroneus brevis tendon (black asterisk); Ca, calcaneus.

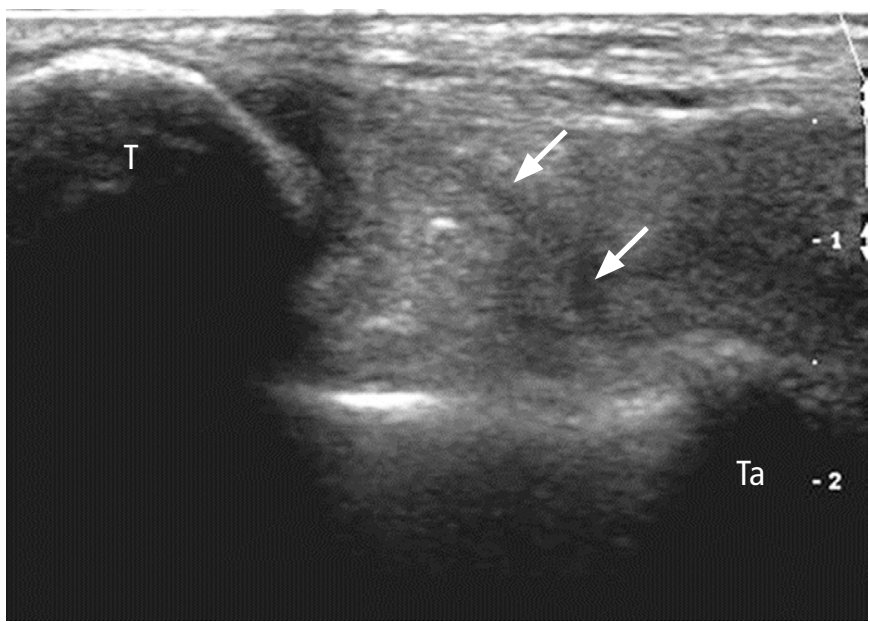

A

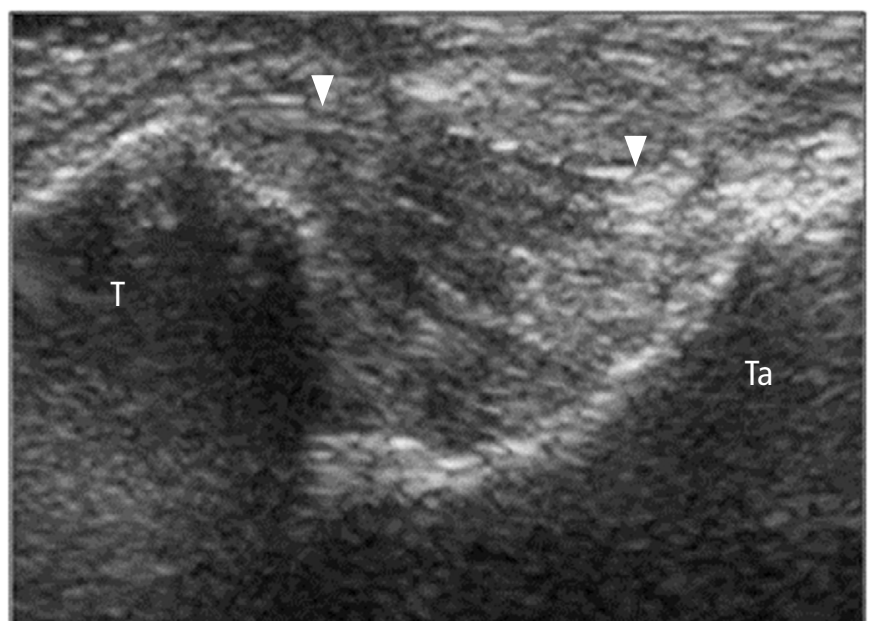

B

Fig. 12. Complete tear of the deltoid ligament.

On the long-axis ultrasonography of a 38-year-old woman with an eversion injury, the deltoid ligament appears swollen with redundant ligament fibers (arrows in A), and suggests a complete tear when compared with a normal fan shape (arrowheads in B) of the contralateral deltoid ligament. T, tibia; Ta, talus. 
pain in the toes and the soles of the feet.

The causes of tarsal tunnel syndrome can be divided into intrinsic and extrinsic factors, or a combination thereof. Intrinsic factors include space-occupying lesions (such as a ganglion cyst, a lipoma, enlarged veins, a tumor, or a neuroma), an osteophyte, hypertrophic retinaculum, tendinopathy, and adhesion or fibrosis secondary to hemorrhage. Extrinsic factors include direct trauma, foot deformities (varus or valgus heel), generalized lower limb edema, constrictive footwear, and systemic inflammatory arthropathy $[35,36]$. In the Japanese literature, the causes of tarsal tunnel syndrome are well documented, with the ganglion and talocalcaneal coalition being prominent [37]. Similarly, another study reported that the ganglion and talocalcaneal coalition represented $40 \%-50 \%$ of the cause of tarsal tunnel syndrome [38].

The ganglion is usually depicted as an anechoic or hypoechoic area with a round or multilobular shape (Fig. 14). Some ganglia are associated with talocalcaneal coalitions [37]. If a ganglion is suspected, a description of the size, location, morphology, and relationship between the posterior tibial artery and the ganglion is recommended [39].

To diagnose talocalcaneal coalition, radiography is essential to show the bony prominence inferior to the medial malleolus. US can

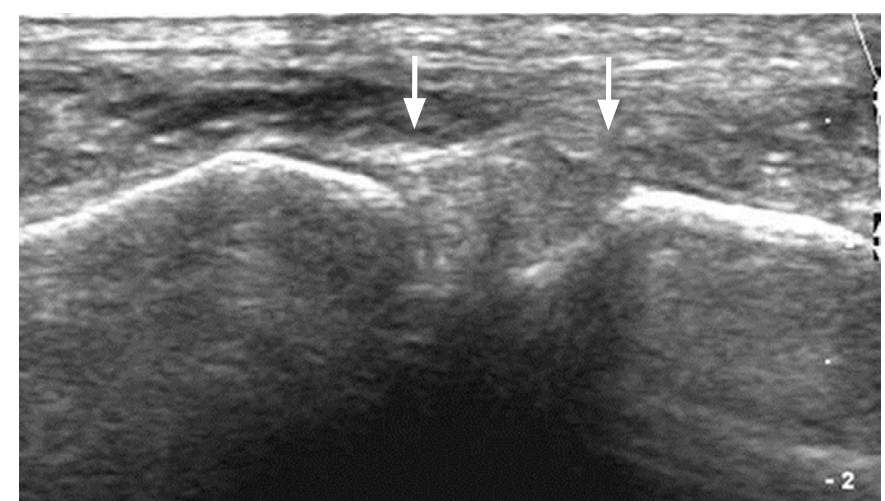

A

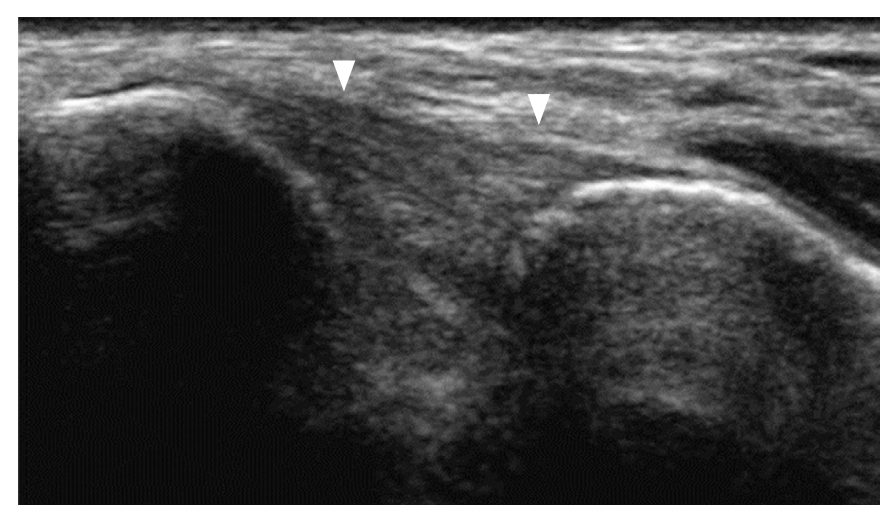

B also reveal a beak-shaped bony process in short-axis images (Fig. 15) [39].

The tibial nerve passes through the medial side of the ankle and branches into the medial plantar nerve, lateral plantar nerve, and medial calcaneal branch. This area is called the tarsal tunnel. These components are covered by the flexor retinaculum from the medial malleolus of the tibia, and slight changes of the calcaneus in this space can easily result in neuropathy (Figs. 14, 15). Dynamic analysis with US can contribute to the diagnosis by revealing small lesions, arterial pulsation, space-occupying lesions, and nerves.

\section{Lipomatosis of the Nerve}

Lipomatosis of the nerve, which is also known as fibrolipomatous hamartoma, is a rare benign tumor-like process characterized by infiltration of the epineurium by adipose and fibrous tissues leading to nerve enlargement [40]. It has a high prevalence in the upper extremities, and most cases involve the median nerve, although other nerves can be involved [41]. In this condition, fatty tissue exists between the nerve fascicles, similar to a coaxial cable in the axial plane, and a spaghetti-like appearance can be observed in the coronal plane (Fig. 16) [42].

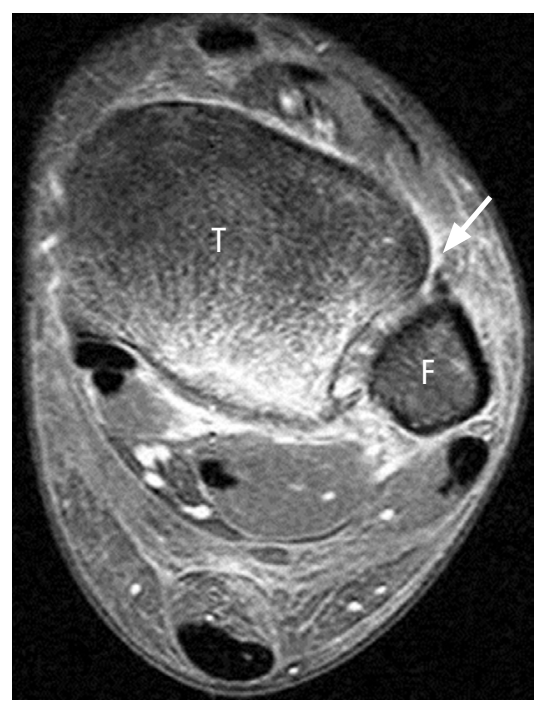

C

Fig. 13. Complete tear of the AiTFL.

A. Long-axis ultrasonography of the AiTFL shows a loss of normal fibrillar continuity with heterogeneous echogenicity (arrows). This finding reveals a complete tear. B. Ultrasonography of opposite ankle shows a normal fibrillar appearance (arrowheads) of the AiTFL compared with a complete tear (A). C. An axial fat-suppressed T2-weighted magnetic resonance image shows a disruption of the AiTFL (arrow) with adjacent soft tissue hyperintensity and bone contusion of posterior malleolus. AiTFL, anterioinferior tibiofibular ligament; $F$, fibula; $T$, tibia. 


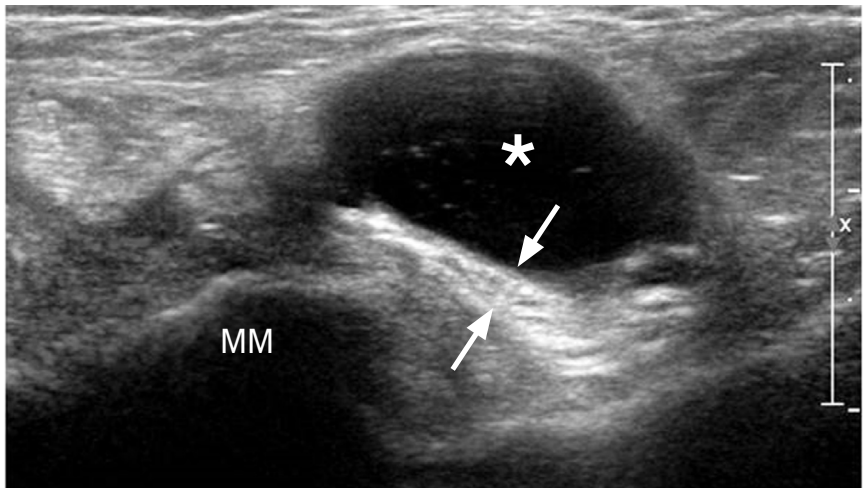

A

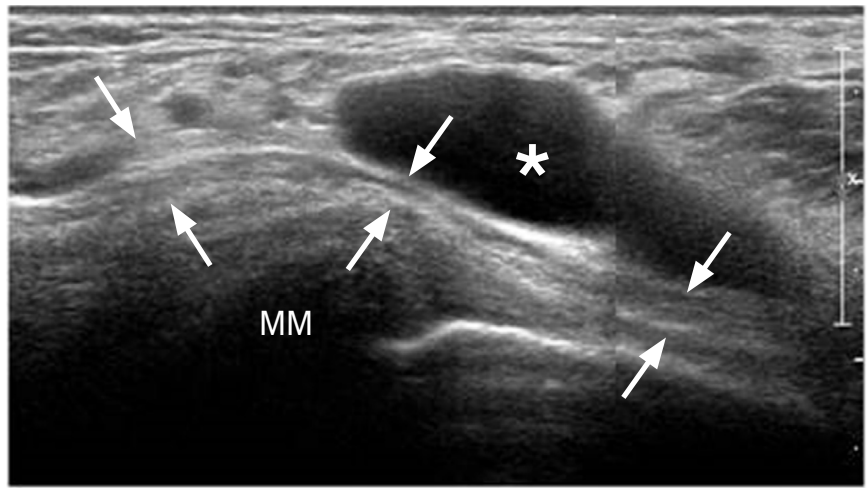

B

Fig. 14. Tarsal tunnel syndrome caused by a ganglion.

Short-axis (A) and long-axis (B) ultrasonography over the medial malleolus shows the compression of the tibial nerve (arrows) by a ganglion (asterisks) in the tarsal tunnel. MM, medial malleolus.

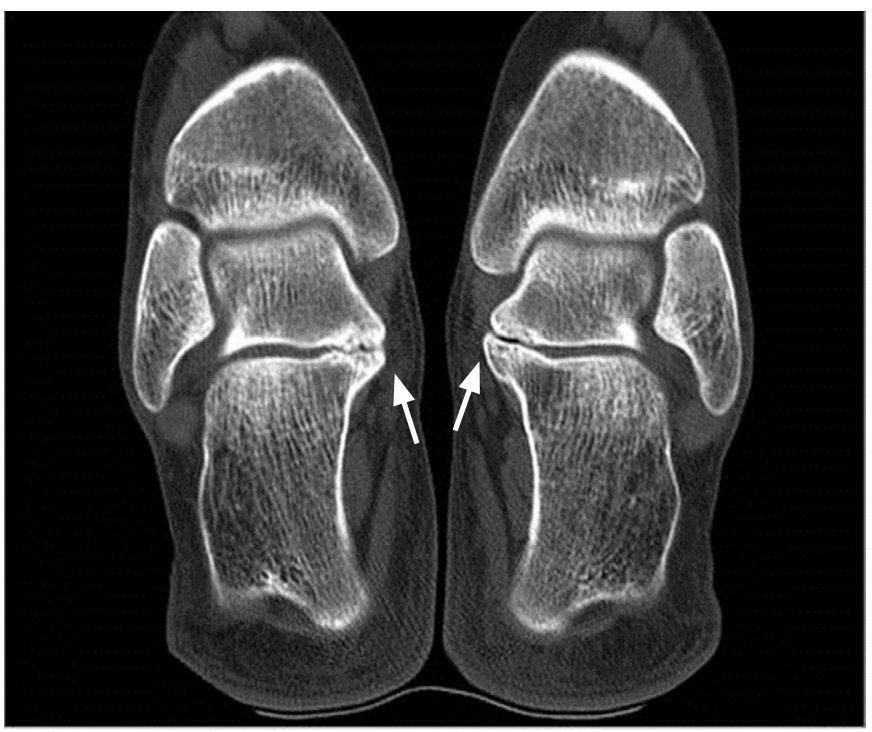

A

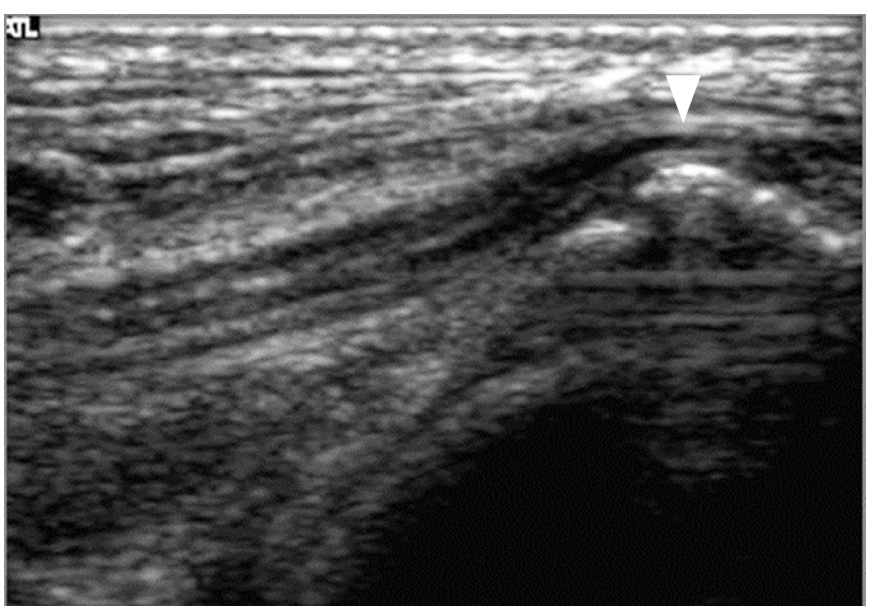

B
Fig. 15. Tarsal tunnel syndrome caused by tarsal coalition.

A. Coronal computed tomography image of both feet demonstrates a fibrous or cartilaginous coalition between the talus and calcaneus (arrows). B, C. Long-axis (B) and short-axis (C) ultrasonography of the tibial nerve shows compression (arrowhead in B) by a bony prominence and swelling of the proximal nerve (arrowhead in C). 


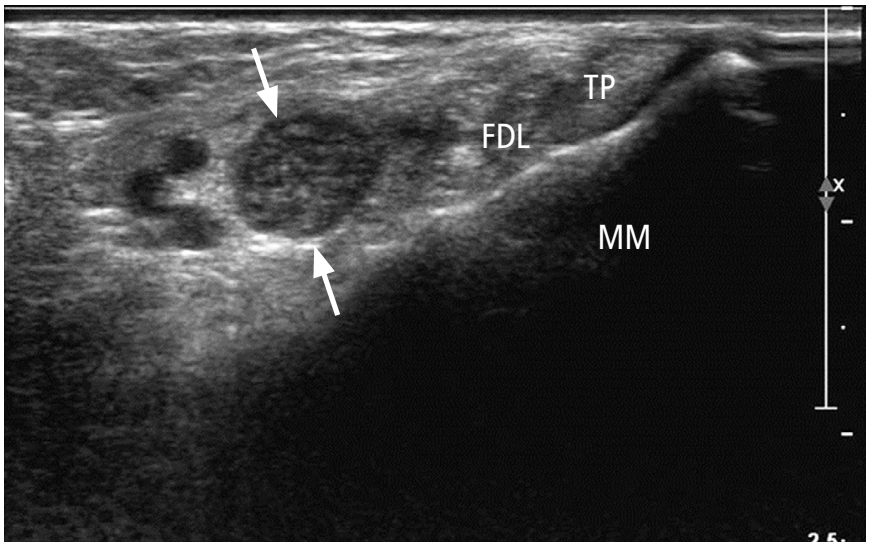

A

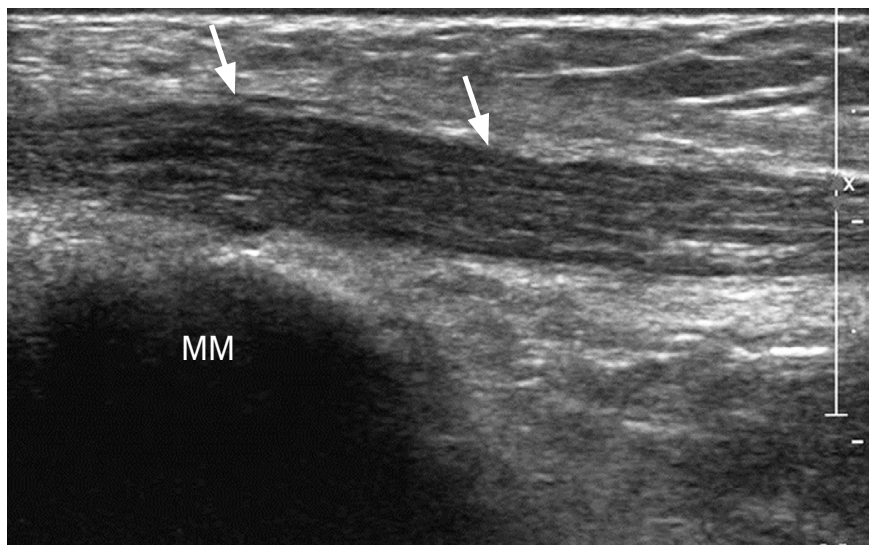

B

Fig. 16. Lipomatosis of the tibial nerve.

Short-axis (A) and long-axis (B) ultrasonography over the medial malleolus shows a thickened and enlarged nerve (arrows), which resembles a thin spaghetti noodle. MM, medial malleolus; TP, tibialis posterior tendon; FDL, flexor digitorum longus tendon.

\section{Conclusion}

The differential diagnosis of ankle pain is extensive. Ankle disorders can be traumatic, inflammatory, infectious, or degenerative. US is the first-line imaging technique used for the detection of lesions in the tendons, ligaments, and nerves of the ankle.

Moreover, dynamic US with a dorsiflexion-eversion maneuver of the ankle may be helpful for the detection of intermittent dislocation and intrasheath subluxation of the peroneal tendons. A stress test during US provides diagnostic confidence regarding the differentiation between partial and complete tears of the ligaments. To identify ankle lesions more accurately, we need to improve our understanding of the normal anatomy of the ankle and to recognize the sonographic features of common pathological conditions affecting this joint.

ORCID: Jung Won Park: http://orcid.org/0000-0003-2636-6417; Sun Joo Lee: http:// orcid.org/0000-0001-6210-9720; Hye Jung Choo: http://orcid.org/0000-0003-39416989; Sung Kwan Kim: http://orcid.org/0000-0001-9459-3772; Heui-Chul Gwak: http://orcid.org/0000-0003-1062-0580; Sung-Moon Lee: http://orcid.org/0000-00022675-3209

\section{Conflict of Interest}

No potential conflict of interest relevant to this article was reported.

\section{Acknowledgments}

This work was supported by a grant from the University Research Park Project of Busan National University funded by Busan Institute of S\&T Evaluation and Planning.

\section{References}

1. Fong DT, Man CY, Yung PS, Cheung SY, Chan KM. Sport-related ankle injuries attending an accident and emergency department. Injury 2008;39:1222-1227.

2. De Maeseneer M, Marcelis $S$, Jager T, Shahabpour M, Van Roy $\mathrm{P}$, Weaver J, et al. Sonography of the normal ankle: a target approach using skeletal reference points. AJR Am J Roentgenol 2009;192:487-495.

3. Bianchi S, Martinoli C, Gaignot C, De Gautard R, Meyer JM. Ultrasound of the ankle: anatomy of the tendons, bursae, and ligaments. Semin Musculoskelet Radiol 2005;9:243-259.

4. Khoury V, Guillin R, Dhanju J, Cardinal E. Ultrasound of ankle and foot: overuse and sports injuries. Semin Musculoskelet Radiol 2007;11:149-161.

5. Hodgson RJ, O'Connor PJ, Grainger AJ. Tendon and ligament imaging. Br J Radiol 2012;85:1157-1172.

6. Neustadter J, Raikin SM, Nazarian LN. Dynamic sonographic evaluation of peroneal tendon subluxation. AJR Am J Roentgenol 2004;183:985-988.

7. Lee SJ, Jacobson JA, Kim SM, Fessell D, Jiang Y, Dong Q, et al. Ultrasound and MRI of the peroneal tendons and associated pathology. Skeletal Radiol 2013;42:1191-1200.

8. Rosenberg ZS, Beltran J, Bencardino JT. From the RSNA Refresher Courses. Radiological Society of North America. MR imaging of the ankle and foot. Radiographics 2000;20 Spec No:S153-S179.

9. Boonthathip M, Chen L, Trudell D, Resnick D. Lateral ankle ligaments: MR arthrography with anatomic correlation in cadavers. Clin Imaging 2011;35:42-48.

10. Jacobson JA, van Holsbeeck MT. Musculoskeletal ultrasonography. Orthop Clin North Am 1998;29:135-167.

11. Nazarian LN, Rawool NM, Martin CE, Schweitzer ME. Synovial fluid 
in the hindfoot and ankle: detection of amount and distribution with US. Radiology 1995;197:275-278.

12. Grant TH, Kelikian AS, Jereb SE, McCarthy RJ. Ultrasound diagnosis of peroneal tendon tears: a surgical correlation. J Bone Joint Surg Am 2005;87:1788-1794.

13. Bianchi S, Delmi M, Molini L. Ultrasound of peroneal tendons. Semin Musculoskelet Radiol 2010;14:292-306.

14. Bencardino JT, Rosenberg ZS, Serrano LF. MR imaging features of diseases of the peroneal tendons. Magn Reson Imaging Clin N Am 2001;9:493-505.

15. Philbin TM, Landis GS, Smith B. Peroneal tendon injuries. J Am Acad Orthop Surg 2009;17:306-317.

16. Raikin SM, Elias I, Nazarian LN. Intrasheath subluxation of the peroneal tendons. J Bone Joint Surg Am 2008;90:992-999.

17. Butler BW, Lanthier J, Wertheimer SJ. Subluxing peroneals: a review of the literature and case report. J Foot Ankle Surg 1993;32:134139.

18. Davis WH, Sobel M, Deland J, Bohne WH, Patel MB. The superior peroneal retinaculum: an anatomic study. Foot Ankle Int 1994; 15:271-275.

19. Oden RR. Tendon injuries about the ankle resulting from skiing. Clin Orthop Relat Res 1987;(216):63-69.

20. Jarvinen TA, Kannus P, Maffulli N, Khan KM. Achilles tendon disorders: etiology and epidemiology. Foot Ankle Clin 2005; 10:255266.

21. Maffulli N, Sharma P, Luscombe KL. Achilles tendinopathy: aetiology and management. J R Soc Med 2004;97:472-476.

22. Almekinders LC, Temple JD. Etiology, diagnosis, and treatment of tendonitis: an analysis of the literature. Med Sci Sports Exerc 1998;30:1183-1190.

23. Hartgerink P, Fessell DP, Jacobson JA, van Holsbeeck MT. Full- versus partial-thickness Achilles tendon tears: sonographic accuracy and characterization in 26 cases with surgical correlation. Radiology 2001;220:406-412.

24. Scheller AD, Kasser JR, Quigley TB. Tendon injuries about the ankle. Clin Sports Med 1983;2:631-641.

25. Haglund P. Beitrag zur Klinik der Achillessehne. Z Orthop Chir 1927;49:49-58.

26. Sofka CM, Adler RS, Positano R, Pavlov H, Luchs JS. Haglund's syndrome: diagnosis and treatment using sonography. HSS J 2006;2:27-29.
27. Rodriguez CP, Goyal M, Wasdahl DA. Best cases from the AFIP: atypical imaging features of bilateral Achilles tendon xanthomatosis. Radiographics 2008;28:2064-2068.

28. Peetrons P, Creteur V, Bacq C. Sonography of ankle ligaments. J Clin Ultrasound 2004;32:491-499.

29. Morvan G, Mathieu P, Busson J, Wybier M. Ultrasonography of tendons and ligaments of foot and ankle. J Radiol 2000;81:361380.

30. Kumai T, Takakura Y, Rufai A, Milz S, Benjamin M. The functional anatomy of the human anterior talofibular ligament in relation to ankle sprains. J Anat 2002;200:457-465.

31. van den Bekerom MP, Oostra RJ, Golano P, van Dijk CN. The anatomy in relation to injury of the lateral collateral ligaments of the ankle: a current concepts review. Clin Anat 2008;21:619-626.

32. Lagalla R, lovane A, Midiri M, Lo Casto A, De Maria M. Comparison of echography and magnetic resonance in sprains of the external compartment of the ankle. Radiol Med 1994;88:742-748.

33. Puffer JC. The sprained ankle. Clin Cornerstone 2001;3:38-49.

34. Takao M, Ochi M, Oae K, Naito K, Uchio Y. Diagnosis of a tear of the tibiofibular syndesmosis: the role of arthroscopy of the ankle. J Bone Joint Surg Br 2003;85:324-329.

35. Havel PE, Ebraheim NA, Clark SE, Jackson WT, DiDio L. Tibial nerve branching in the tarsal tunnel. Foot Ankle 1988;9:117-119.

36. Ahmad M, Tsang K, Mackenney PJ, Adedapo AO. Tarsal tunnel syndrome: a literature review. Foot Ankle Surg 2012;18:149-152.

37. Nagaoka M, Satou K. Tarsal tunnel syndrome caused by ganglia. J Bone Joint Surg Br 1999;81:607-610.

38. Takakura Y, Kitada C, Sugimoto K, Tanaka Y, Tamai S. Tarsal tunnel syndrome: causes and results of operative treatment. J Bone Joint Surg Br 1991;73:125-128.

39. Nagaoka M, Matsuzaki H. Ultrasonography in tarsal tunnel syndrome. J Ultrasound Med 2005;24:1035-1040.

40. Mason ML. Presentation of cases: Proceedings of the American Society for Surgery of the Hand. J Bone Joint Surg Am 1953;35:273275.

41. Jain TP, Srivastava DN, Mittal R, Gamanagatti S. Fibrolipomatous hamartoma of median nerve. Australas Radiol 2007;51 Spec No.:B98-B100.

42. Lee $\mathrm{CH}$, Wu JS, Goldsmith JD, Kung JW. Lipomatosis of the sciatic nerve secondary to compression by a desmoid tumor. Skeletal Radiol 2013;42:1751-1754. 